\title{
A portal extension of the standard model with an unbroken local dark $U(1)$
}

\author{
Seungwon Baek, Pyungwon Ko and Wan-II Park* \\ Korea Institute for Advanced Study \\ E-mail: sbaek1560@gmail.com, pko@kias.re.kr, wipark@kias.re.kr
}

\begin{abstract}
Assuming dark matter is absolutely stable due to unbroken dark gauge symmetry and singlet operators are portals to the dark sector, we present a simple extension of the standard seesaw model that can accommodate all the cosmological observations as well as terrestrial experiments available as of now, including leptogenesis, extra dark radiation of $\sim 8 \%$ (resulting in $N_{\text {eff }}=$ 3.130 the effective number of neutrino species), Higgs inflation, small and large scale structure formation, and current relic density of scalar dark matter $(X)$. The Higgs signal strength is equal to one as in the standard model.
\end{abstract}

The European Physical Society Conference on High Energy Physics -EPS-HEP2013

18-24 July 2013

Stockholm, Sweden

\footnotetext{
${ }^{*}$ Speaker.
} 


\section{Introduction}

The standard model (SM) based on $S U(3)_{C} \times S U(2)_{L} \times U(1)_{Y}$ is confirmed at quantum level with high accuracy. Still the SM has to be extended in order to accommodate the following observations: (i) neutrino masses and mixings, (ii) baryogenesis, (iii) nonbaryonic cold dark matter of the universe, and (iv) inflation and density perturbation.

Paying attention to the stability of dark matter, we notice that an ad hoc $Z_{2}$ symmetry, which is often assumed to stabilize DM in nonsupersymmetric dark matter models, is broken by quantum gravity effects and allows $1 / M_{\text {Planck }}$ suppressed nonrenormalizable operators. So, we are led to local dark symmetry and dark gauge force for the stability of weak scale dark matter. This would be a very natural route for the DM model building, since the unsurpassed successful SM is also based on local gauge symmetry and its spontaneous breaking.

In this talk, we present a simple renormalizable model where the dark matter lives in a dark (hidden) sector with its own dark gauge charge along with dark gauge force. Allowing all the SM singlet portal interactions (provided by Higgs bilinear, right-handed neutrinos and kinetic mixing), we show that all the phenomenological shortcomings of SM can be addressed in the model. This talk is based on Ref. [1], to which we invite the readers for more detailed discussions on the subjects described in this talk.

\section{Model}

We assume that dark matter lives in a hidden sector having an unbroken local $U(1)_{X}$ dark gauge symmetry. All the SM fields are taken to be $U(1)_{X}$ singlets. With these assumptions and all the singlet portal interactions, we can write the most general renormalizable Lagrangian as follows:

$$
\mathscr{L}=\mathscr{L}_{\mathrm{SM}}+\mathscr{L}_{X}+\mathscr{L}_{\psi}+\mathscr{L}_{\text {portal }}+\mathscr{L}_{\text {inflation }}
$$

where $\mathscr{L}_{\mathrm{SM}}$ is the standard model Lagrangian and

$$
\begin{aligned}
\mathscr{L}_{X} & =\left|\left(\partial_{\mu}+i g_{X} q_{X} \hat{B}_{\mu}^{\prime}\right) X\right|^{2}-\frac{1}{4} \hat{B}_{\mu v}^{\prime} \hat{B}^{\prime \mu v}-m_{X}^{2} X^{\dagger} X-\frac{1}{4} \lambda_{X}\left(X^{\dagger} X\right)^{2} \\
\mathscr{L}_{\psi} & =i \bar{\psi} \gamma^{\mu}\left(\partial_{\mu}+i g_{X} q_{X} \hat{B}_{\mu}^{\prime}\right) \psi-m_{\psi} \bar{\psi} \psi \\
\mathscr{L}_{\text {portal }} & =-\frac{1}{2} \sin \varepsilon \hat{B}_{\mu v}^{\prime} \hat{B}^{\mu v}-\frac{1}{2} \lambda_{H X} X^{\dagger} X H^{\dagger} H \\
& -\frac{1}{2} M_{i} \overline{N_{R i}^{C}} N_{R i}+\left[Y_{v}^{i j} \overline{N_{R i}} \ell_{L j} H^{\dagger}+\lambda^{i} \overline{N_{R i}} \psi X^{\dagger}+\text { H.c. }\right] \\
\mathscr{L}_{\text {inflation }} & =\left[\xi_{H} H^{\dagger} H+\xi_{X} X^{\dagger} X\right] R
\end{aligned}
$$

$g_{X}, q_{X}, \hat{B}_{\mu}^{\prime}$ and $\hat{B}_{\mu \nu}^{\prime}$ are the gauge coupling, $U(1)_{X}$ charge, the gauge field and the field strength tensor of the dark $U(1)_{X}$, and $R$ is the scalar curvature, respectively. $\hat{B}_{\mu v}$ is the gauge field strength of the SM $U(1)_{Y}$. We assume $m_{X}^{2}>0, \quad \lambda_{X}>0, \quad \lambda_{H X}>0$, so that the local $U(1)_{X}$ remains unbroken and the scalar potential is bounded from below at tree level.

\section{Implications on particle physics and cosmology}

Our model can accommodate various observations from cosmology and astrophysics with definite predictions for Higgs physics: 
- Dark scalar $X$ can improve the stability of the electroweak vacuum up to Planck scale, unlike the SM for $m_{h} \sim 125 \mathrm{GeV}$ and $m_{t}=173.2 \mathrm{GeV}$ and $\alpha_{s}=0.118$, if $\lambda_{X}>0$ and $\lambda_{H X} \gtrsim 0.2$ [2].

- Perturbativity of quartic couplings for scalar fields $H$ and $X$ up to Planck scale puts theoretical constraints on $\lambda_{X}$ and $\lambda_{H X}$ such that $\lambda_{X} \lesssim 0.2$ and $\lambda_{H X} \lesssim 0.6$ [2].

- Self-interaction of DM mediated by massless dark photon can solve the small scale problem of DM subhalo while satisfying constraints from inner structure and kinematics of dark matter halos, if $g_{X} \lesssim 2.5 \times 10^{-2}\left(m_{X} / 300 \mathrm{GeV}\right)^{3 / 4}$.

- The dark scalar $X$ can be annihilated efficiently thanks to the Higgs portal interaction even if $g_{X}$ is very small.

- Direct detection experiments such as XENON100 put a strong bound, $10^{-12} \lesssim \varepsilon g_{X} \lesssim 10^{-5}$ for $6 \mathrm{GeV} \lesssim m_{X} \lesssim 1 \mathrm{TeV}$ when the upper bound on $g_{X}$ is used.

- We find that dark photon contributes to dark radiation by $\Delta N_{\text {eff }} \sim 0.08$, which is in agreement with the recent Planck data, $N_{\text {eff }}=3.30 \pm 0.27$ at $68 \% \mathrm{CL}$.

- The decay of RH neutrinos and $\psi$ generate large enough lepton number asymmetries to match observation.

- Higgs inflation [3] can work in our model since the gauge singlet scalar coupled to SM Higgs field cures the instability of potential in Higgs-singlet system [4].

- In case $U(1)_{X}$ is unbroken, the Higgs signal strength should be equal to "1", independent of production and decay channels.

In conclusion, we presented a simple extension of the standard seesaw model where dark sector is constructed with local dark gauge symmetry and renormalizability allowing all the SM singlet portal interactions. This model provides possible solutions to all the phenomenological shortcomings of SM, hence it could be considered as an alternative to the new minimal SM [5].

\section{References}

[1] S. Baek, P. Ko and W. -I. Park, Singlet Portal Extensions of the Standard Seesaw Models to a Dark Sector with Local Dark Symmetry, JHEP 1307, 013 (2013) [arXiv:1303.4280 [hep-ph]].

[2] S. Baek, P. Ko, W. -I. Park and E. Senaha, Higgs Portal Vector Dark Matter : Revisited, JHEP 1305, 036 (2013) [arXiv:1212.2131 [hep-ph]].

[3] F. L. Bezrukov and M. Shaposhnikov, The Standard Model Higgs boson as the inflaton, Phys. Lett. B 659, 703 (2008) [arXiv:0710.3755 [hep-th]].

[4] O. Lebedev, On Stability of the Electroweak Vacuum and the Higgs Portal, Eur. Phys. J. C 72, 2058 (2012) [arXiv:1203.0156 [hep-ph]].

[5] H. Davoudiasl, R. Kitano, T. Li and H. Murayama, The New minimal standard model, Phys. Lett. B 609, 117 (2005) [hep-ph/0405097]. 